\title{
Computation and Analysis of Heart Sound Signals using Hilbert Transform and Hilbert-Huang Transform
}

\author{
Mehak Saini ${ }^{1}$, Madhwendra Nath ${ }^{2}$, Priyanshu Tripathi ${ }^{3}$, Dr. Sanju Saini ${ }^{4}$, Dr. K.K. Saini ${ }^{5}$ \\ ${ }^{1,4}$ Deenbandhu Chhoturam University of Science and Technology Murthal, Sonepat, Haryana, India \\ ${ }^{2,3,4}$ Hindu College of Engineering Sonepat, Haryana, INDIA \\ ${ }^{1}$ mhk.saini1904@gmail.com'2 madhwendra.711@gmail.com, \\ 3prk.triapthi@gmail.com, ${ }^{4}$ sanjusaini.ee@dcrustm.org,, ${ }^{5}$ dphce2015@gmail.com
}

\begin{abstract}
Phonocardiograph is a continuous, non-invasive, low cost and accurate recording and monitoring of valve functioning of heart using heart sound signal. When Hilbert Transform is directly applied, an Analytic Signal of Heart Sound Signal is obtained. In order to decipher the details of the signal, Hilbert-Huang Transform is applied on the Heart Sound Signal. The HHT has two step analyses: The first one is Empirical Mode Decomposition (EMD) and the second part is Hilbert Spectral Analysis (HSA). The HHT analysis has been applied for pre-exercise and post-exercise data acquired from a subject. The subject had to go for stair-exercise (75 stepped stairs) for this purpose.
\end{abstract}

Keyword- Hilbert Transform, HHT, intrinsic mode functions, Empirical Mode Decomposition, Hilbert Spectrum

\section{INTRODUCTION}

Signal processing is most growing and a area having emerging technology now a days. Signal processors are sometimes used for radio, transportation, medicine and production field etc. In 1743 a renowned Swiss mathematician Leonard Euler (1707-1783) derived the formula:

$$
\mathrm{e}^{\mathrm{jz}}=\cos (\mathrm{z})+\mathrm{j} \sin (\mathrm{z}) \quad \ldots
$$

After 150 years, physicist Arthur E. Kennelly and the scientist Charles P. Steinmetz used this formula to derive the complex notation for harmonic wave forms in electrical engineering as follows,

$$
\mathrm{e}^{\mathrm{j} \omega \mathrm{t}}=\cos (\omega \mathrm{t})+\mathrm{j} \sin (\omega \mathrm{t}) \quad \ldots \ldots
$$

Later on, in starting of 20th century, the German scientist David Hilbert (1862- 1943) proved that the function $\sin (\omega t)$ is simply Hilbert transform of $\cos (\omega t)$. This produced the $\pm \Pi / 2$ phase-shift operator which is an elementary property of the Hilbert transform.

\subsection{Hilbert Transform}

In time domain, The Hilbert transform of any function $y(t)$ is defined for all $t$ by,

$$
\hat{y}(t)=\frac{1}{n} P \int_{-\infty}^{+\infty} \frac{y(\tau)}{(t-\tau)} d t \quad \ldots .
$$

Where, the $\mathrm{P}$ in front of the integral denotes the Cauchy principal value which expands the class of functions for which the integral exist [1]. In other words Hilbert transform of an incoming signal may be defined as a convolution between the signal and the Hilbert transformer $\mathrm{h}(\mathrm{t})$, i.e.

$$
\hat{\mathrm{y}}(\mathrm{t})=\mathrm{h}(\mathrm{t}) * \mathrm{y}(\mathrm{t}) \quad \ldots \ldots
$$

Where * represents convolution;

$\hat{y}(t)=$ The Hilbert Transform of $y(t)$;

$h(t)=$ The time impulse response of Hilbert transformer $[h(t)=1 /(n t)]$;

$y(t)=$ A real continuous time domain input. 
When talking of the frequency domain Hilbert transform may be defined as phase shift of $\pm \Pi / 2$ over the frequency range of the signal.

\subsection{Hilbert-Huang Transform: An Ideal Tool for PCG analysis:}

The development of the Hilbert-Huang Transform (HHT) was inspired by the need to describe non-linear, complex and distorted type of waveforms [4]. Following points can prove that HHT is ideal tool for Heart Sound (PCG) analysis:

$>$ The Traditional data-analysis methods are based on stationary and linear assumptions.

> Since, in most real systems, either natural or even man-made ones, the data are found to be both nonlinear and non-stationary, mostly.

> A necessary condition to represent nonlinear and non-stationary data is that it should be adaptive.

$>$ This method is adaptive which means the definition of the basis is data-dependent i.e. a posterior-defined basis.

> The HHT method is potentially much suitable for non-stationary, non-linear and especially for timefrequency-energy representation of data.

Since, Heart Sound (PCG) is also a non-stationary and non-linear signal. Hence, HHT is ideal tool for heart sound (PCG) analysis.

\subsection{Huang-Hilbert Transforms (Modified Hilbert Transform):}

This new method (HHT) has been developed for the analysis of nonlinear and non-stationary data. The important part of this method is "Empirical mode decomposition" i.e. EMD. Using EMD, any complex and complicated data could be decomposed into a finite and small number of "intrinsic mode functions" i.e., IMFs. These IMFs admit well-behaved Hilbert transforms. Such kind of decomposition method is much adaptive and highly efficient. As this decomposition method is completely based on the some local characteristic of time scale of the data, therefore, it's applicable to nonlinear as well as non-stationary processes. The implementation of the Hilbert transform on obtained IMFs, yield instantaneous frequencies as functions of time. These instantaneous frequencies are having sharp identifications of imbedded information. The final result is presented in the form of energy-frequency-time distribution, which is also called as Hilbert Spectrum. In this complete method, the central theme is the introduction of the "intrinsic mode functions" that is based on local characteristics of the signal, which makes the instantaneous Frequency much informative [5],[19],[20].

\subsection{Hilbert Spectrum:}

After obtaining the components of intrinsic mode function, it would be easier to apply the Hilbert transform to each IMFs followed by computation of the instantaneous frequency. By performing the Hilbert transform on each IMF, the data can be expressed in following form:

$$
\mathrm{x}(\mathrm{t})=\sum_{\mathrm{i}=0}^{\mathrm{n}} \mathrm{a}_{\mathrm{j}}(\mathrm{t}) \cdot \mathrm{e}^{\left(\mathrm{i} \int \omega_{\mathrm{j}}(\mathrm{t}) \cdot \mathrm{dt}\right)} \quad \ldots \ldots
$$

Above equation is also useful for representation of amplitude and instantaneous frequency as functions of time in a 3-D plot, in which the amplitude can be contoured on the plane of frequency-time. This frequency-time distribution of the amplitude is called as the Hilbert amplitude spectrum, $\mathrm{H}(\omega, \mathrm{t})$, i.e. "Hilbert spectrum". 


\section{IMPLEMENTATION}

\subsection{Implementation of Hilbert Transform on PCG signal:}

The platform used to implement Hilbert transform to the Heart Sound (PCG) signal is MATLAB 7.4.

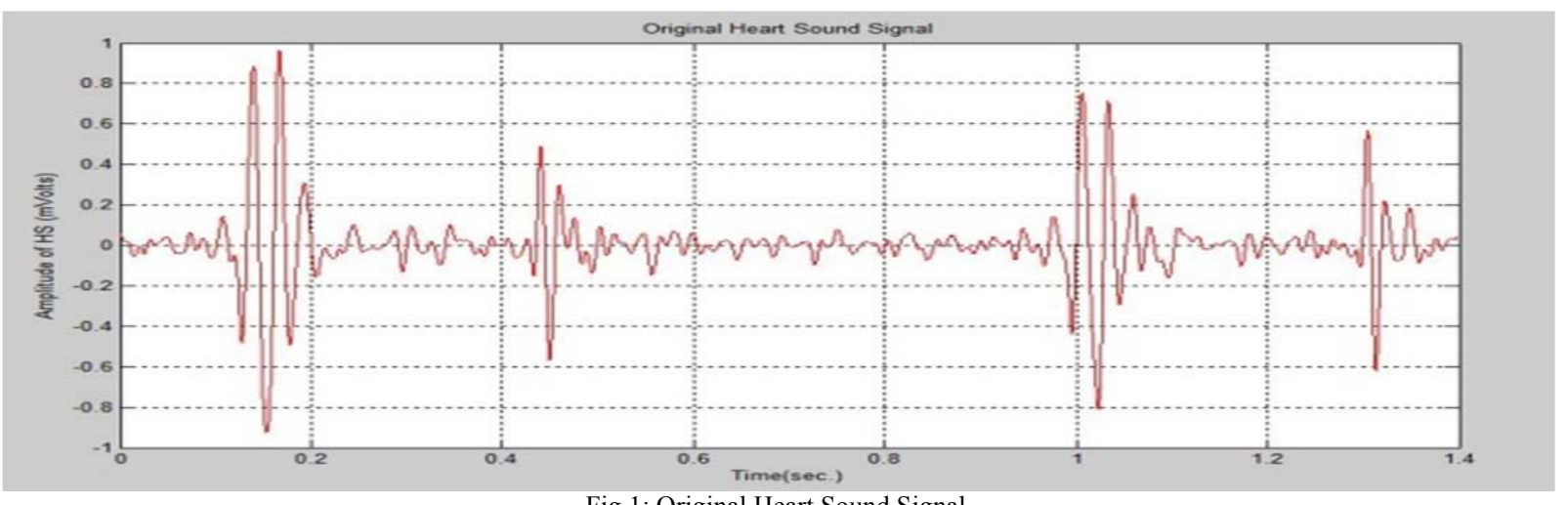

Fig.1: Original Heart Sound Signal

As far as Hilbert Transform is concerned there is an in built function of finding Hilbert in MATLAB. Such that if $\mathrm{x}$ is the array which stores the samples of the Heart sound (PCG) signal. And $\mathrm{y}$ is an array that stores complex samples where the real part is given by the corresponding sample in the array $\mathrm{x}$ and the imaginary value is that of the Hilbert transform of signal. In other words $\mathrm{y}$ is the analytic signal formed by the Hilbert

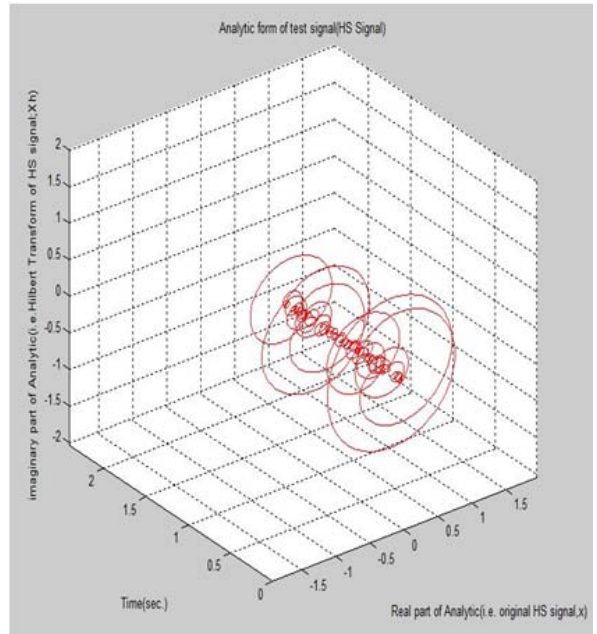

Fig.2: Analytic signal of Heart Sound Signal

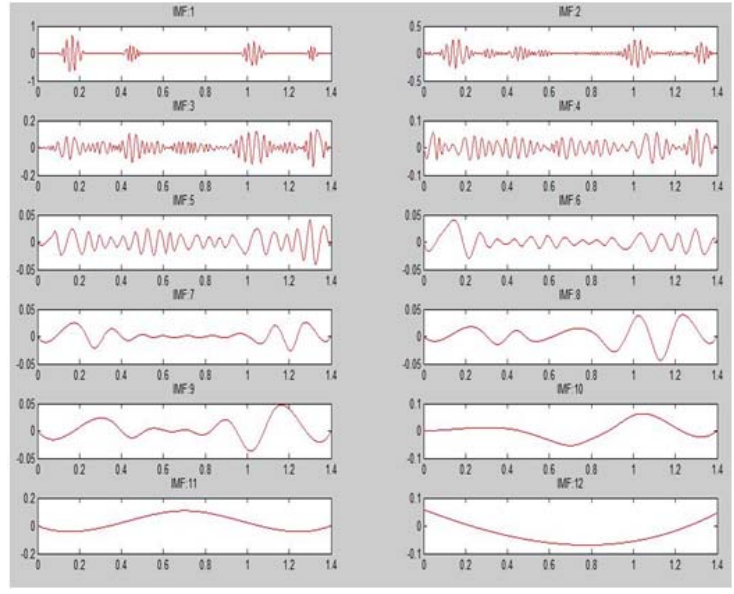

Fig.3: Total 12 IMFs are obtained after decomposition of Heart Sound Signal of a subject in laying situation (Before exercise)

transform of the signal $\mathrm{x}$.

Here, the Fig. 1 shows the original Heart Sound Signal, which is taken for analysis. The Fig. 2 shows the Analytic signal of original Heart Sound signal. The analytic representation is a generalization of the phasor form of waveform. After making Heart Sound signal Analytic, we can look it as a rotating vector having instantaneous phase $\Phi(\mathrm{t})$ and instantaneous amplitude $\mathrm{A}(\mathrm{t})$ in the time domain. Since the any signal and its Hilbert transform are always mutually orthogonal (i.e. phase shift occurs by $\pi / 2$ ), hence, the analytic seems to be in "Spiral shape" (as in Fig.2). Hence, the finally obtained Analytic signal is superimposition of original signal and its Hilbert Transform with respect to time axis which is clearly visible by its three dimensional plot.

\subsection{IMPLEMENTATION OF HILBERT-HUANG TRANSFORM ON HEART SOUND SignAL}

Since Heart Sound (PCG) signal is a non-stationary \& non-linear signal so directly applying Hilbert transform to the Heart Sound signal to find instantaneous frequency is of low significance. Hence we need to decompose the Heart Sound signal into simpler Intrinsic Mode Functions (IMFs) so that we can minutely 
identify and analyze the frequency contents of the signal. As discussed earlier that the Hilbert-Huang Transform analysis consists of two section:

a) Empirical Mode Decomposition (EMD) of Signal, in which we decompose the original Heart Sound Signal into IMFs.

b) Hilbert Spectral Analysis (HSA), in which we apply the Hilbert transform on the different components (IMFs), obtained after decomposition of original HS signal and find out the Hilbert Spectrum (i.e. EnergyFrequency-Time plot) finally.

Empirical Mode Decomposition of Heart Sound (PCG) Signal:

As shown in Fig.3, the Empirical Mode Decomposition is applied on original Heart Sound Signal to obtain Intrinsic Mode Functions. Total 12 IMFs are obtained after decomposition. The twelfth IMFs is not an IMF, it's a residue which is monotonic function.

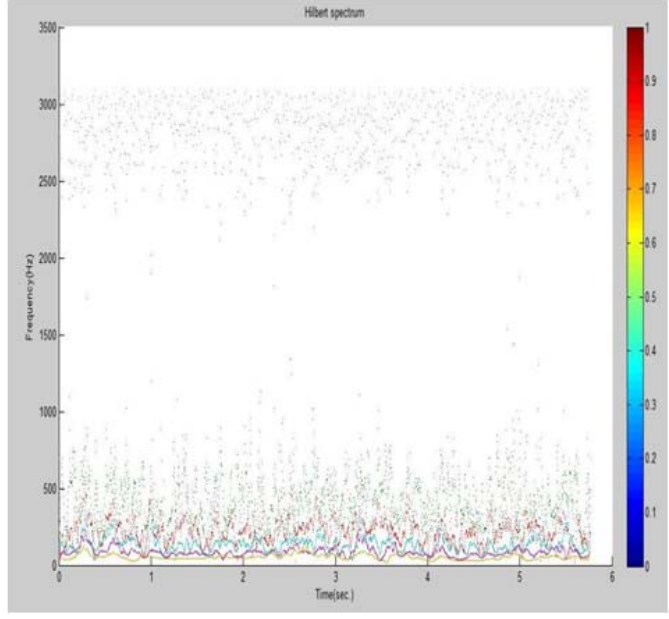

Fig.4: Hilbert Spectrum of Heart Sound Signal of a subject (the heart sound data taken in laying position)

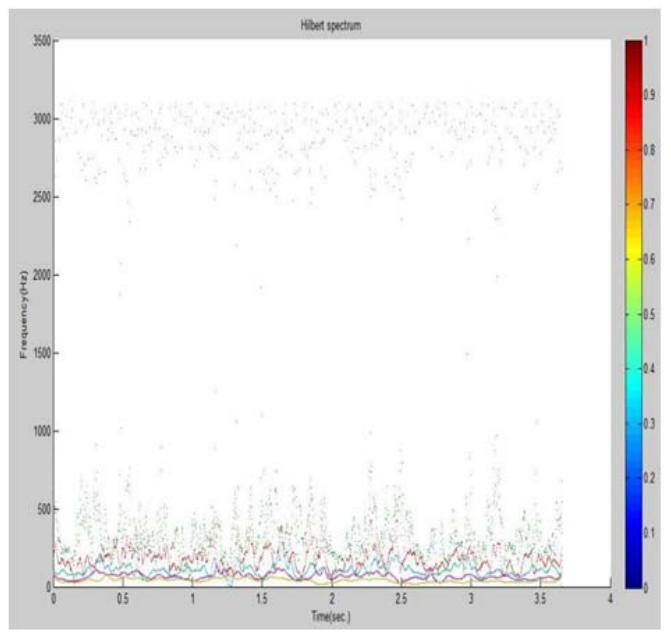

Fig.5(b): Energy-Frequency-Time plot (Hilbert Spectrum) of heart sound of a subject(in laying situation, postexercise)

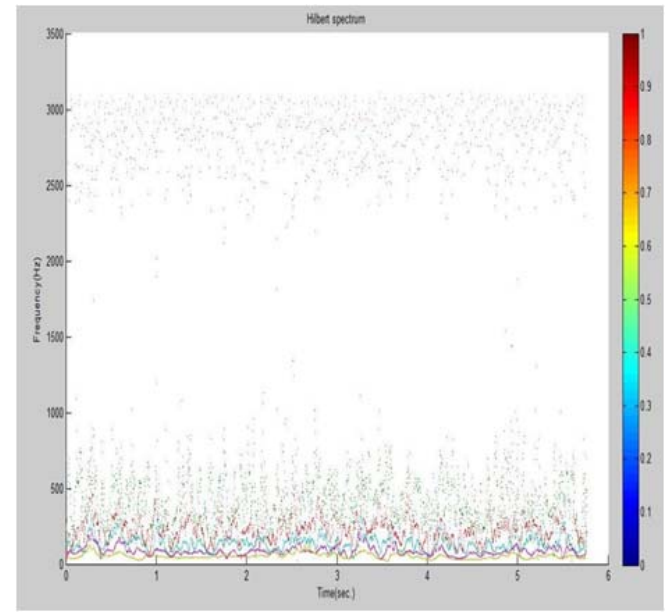

Fig.5(a): Energy-Frequency-Time plot (Hilbert Spectrum) of heart sound of a subject(in laying situation, pre-exercise)

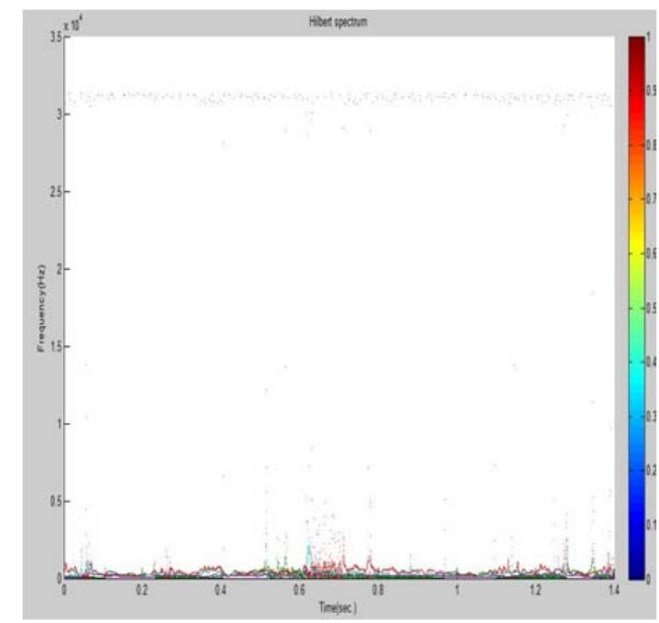

Fig.6(a): Hilbert Spectrum of heart sound of a normal subject (Age group 19-28, in laying situation)

\section{Applications of Hilbert Transform to IMFs after EMD:}

Since we have decomposed the Original Heart Sound signal and got the IMFs. Now after applying the Hilbert Transform on IMFs obtained, we can easily analyse that which frequencies occurred and when during the signal duration, and also shows at a glance that where most of the signal energy is concentrated in time and frequency space. Hence in this particular case (which is shown in Fig.3) we can get 12 analytic signals after 
applying the Hilbert Transform on each IMF, from which we can find the instantaneous frequency profile of each IMF.

\section{Construction of Hilbert Spectrum:}

As it is described earlier that by construction of Hilbert Spectrum one can easily analyse that which frequencies occurred and when, during the signal duration, and it also shows at a glance that where most of the signal energy is concentrated in the time and the frequency space. Hence, In Hilbert Spectrum, a 3-D plot may be constructed of the amplitude at a given time and frequency.

The final Hilbert spectrum which is formed from the Huang-Hilbert Transform is shown in Fig 5 and Fig 6. Where the colour of each frequency component describes the extent of energy it contributes to the main the input signal hence giving a view of the frequency content of the signal at each instant of the input signal. The near the colour to red the more that frequency contributes to that signal and the nearer to blue it is lesser the contribution. This is coloured scattered plot.

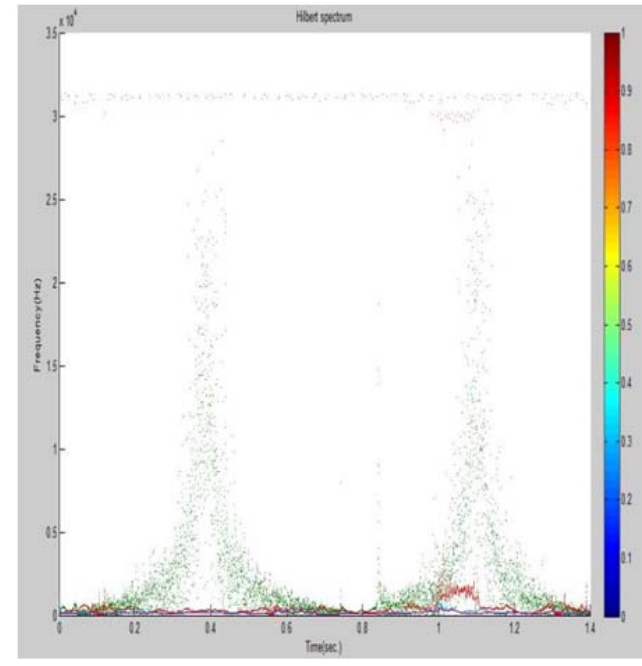

Fig.6(b): Hilbert Spectrum of heart sound of an aged subject (Age group 76-87, in laying situation)

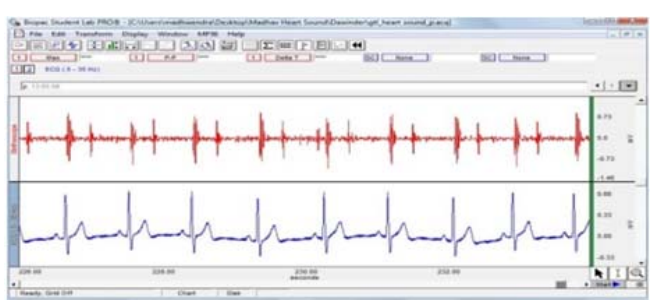

Fig.7(a): Heart Sound and ECG of a normal subject (Age group 19-28 years)

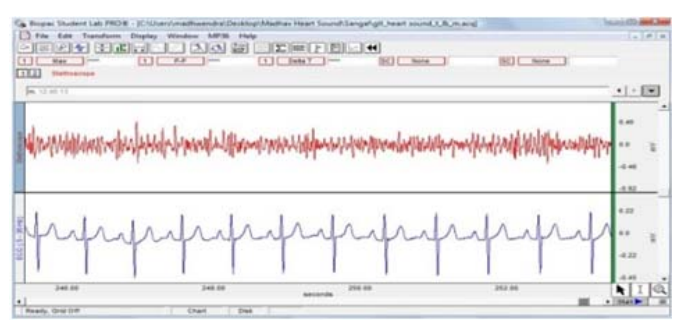

Fig.7(b): Heart Sound and ECG of a normal subject (Age group 76-87 years)

\section{RESUlTS AND CONCLUSIONS}

Now, the Hilbert-Huang Transform is applied to the Heart Sound data. Through this analysis we intend to find the effects of Postural and situation changes on the Heart Sound data of the subject. Therefore first we consider a healthy subject and record the Heart Sound (PCG) while the subject is in lying position and relaxed stage. Then, we record the subject's data (i.e. pre-exercise data) and apply the Hilbert-Huang Transform to find the Energy-Frequency-Time plot (Hilbert Spectrum). After this we make the subject to have stair-exercise (to go upstairs and downstairs through 75 steps stairs). Then, we repeat the above whole procedure to find the EnergyFrequency-Time plot for the subject (i.e. post-exercise data analysis). Hence, the obtained two plots are shown in Fig.5

Looking at Hilbert Spectrum in Fig.5 (a), it is evident that, the dominant-energy-frequency band of signal is constituted by the higher frequency (HF) components. Now, observing the the energy spectrum if Fig. 5 (b) we find noticeable change in the instantaneous frequency of the IMFs. Here, we can conclude that, the dominant-energy-frequency band of signal is constituted by lower frequency (LF). This occurs because; respiration signal and a little bit muscles noise create interference with Heart sound signal and makes the dominant-energy-frequency components into lower frequency (LF) region.

Now, a subject of age group 76-87 years has been considered and heart sound has been recorded while subject in the laying position and relaxed stage. Then Huang-Hilbert Transform is applied to find the Hilbert Spectrum of the Heart Sound data of the aged subject. Now, we compare the Hilbert spectrum of this aged subject with that of another healthy subject of age group 19-28 years. The both plots are shown in Fig.6.

Looking at the Hilbert spectrum in Fig.6(a), it is evident that, the dominant-energy-frequency range of signal is constituted by the low frequency (LF) components. And when we observe at the Fig.6(b), we find that, the dominant-energy-frequency range constituted by the higher frequency (HF) components. This information suggests the better situation of heart valves of normal subject of age group (19-28 years) in comparison to that of aged (age group 76-87 years) subject. This frequency variation also suggests some problem in working of 
circulatory system and Heart Valves of aged subject. The original heart sound and ECG signal of normal subject (age group, 19-28 years) and aged subject (age group, 76-87 years) have been shown in Fig.7.

\section{FUTURE SCOPE}

The Hilbert-Huang Transform analysis could be implemented to study minutely the impact on heart sound \& its Hilbert Spectrum due to various postural and subject's situation changes. There is also a future scope to study and distinguish the spectral changes of Heart Sound due to different types of Heart Murmurs e.g. aortic valve stenosis, Aortic Regurgitation, Atrial Septal Defect (ASD), Mitral Regurgitation and Ventricular Septal Defect (VSD) etc.

\section{REFERENCES}

[1] H. Peter, "Applied and computational complex analysis”, Vol. 1, J.Wiley \& Sons, Inc., New York, 1988.

[2] KK Saini, S Saini," a New Architecture for Intelligent Systems with Logic Based Languages", AIP Conference Proceedings, Vol. 1052, issue 1, 2008, pp. 93-101.

[3] Goldberg R. R., "Fourier transforms", Cambridge university press, Cambridge, 1961.

[4] N. E. Huang and Samuel S P Shen, "Hilbert-Huang Transform and its Applications", World Scientific publishing co. Pte. ltd., Vol. 5, pp. 1-24, 2005

[5] N. E. Huang, Z. Shen, S. R. Long,"The empirical mode decomposition and the Hilbert spectrum for nonlinear and nonstationary timeseries analysis", Proceedings of the Royal Society of London A, Vol. 454, no. 1971, pp. 903-995, 1998.

[6] R. M. Rangayyan, "Biomedical Signal Processing. A case study approach”, J. Wiley \& Sons, Inc., New York, 2002.

[7] L. A. Geddes,"Birth of the stethoscope," Engineering in Medicine and Biology Magazine, IEEE, Vol. 24, pp. 84, 2005.

[8] A. N. Pelech,"The physiology of cardiac auscultation", Pediatr Clin North Am., Vol. 51, pp.1515-1535, 2004.

[9] Lehner R. J., R. M. Rangayyan, "A three-channel microcomputer system for segmentation and characterization of the phonocardiogram", IEEE Transactions on Biomedical Engineering,34:485-489,1987.

[10] KK Saini, S Saini,"The application of neural networks with artificial intelligence technique in the modeling of industrial processes", AIP Conference ProceedingsVol. 1052, issue 1, 2008, pp. 87-92.

[11] H. Y. Liang et al,"A heart sound segmentation algorithm using wavelet decomposition and reconstruction," 19th IEEE International Conference on Medicine and Biological Society, USA, Vol. 4, pp. 1630-1633; October 30 - November 2, 1997.

[12] T. Oskiper, R. Watrous, "Detection of the first heart sound using a time-delay neural network," Computers in Cardiology, USA, Vol. 29, pp. 537-540, September 2002.

[13] W. Wang et al, "Analysis of the first heart sound using the matching pursuit method", Medical \& Biological Engineering \& Computing, 39: 644-648, 2001.

[14] J. Herold, et al, "Diagnosing aortic valve stenosis by correlation analysis of wavelet filtered heart sounds", Medical \& Biological Engineering \& Computing, 43: 451-456, 2005.

[15] Bipasha Bhatia, Sanju Saini \& Narender kumar, “Automatic generation control of multi-area power systems using ANN controller", International journal of Engineering Science and Technology, Vol. 4, No. 7, pp. 3329-3334, July 2012.

[16] Mallat S., Zhang Z., "Matching Pursuit with time-frequency dictionaries", IEEE Transactions on Signal Processing, 41:3397-3415, 1993.

[17] Sanju Saini, Arvind kumar, "Speed control of separately excited d.c. motor using self tuned ANFIS techniques", International journal of computer science \& technology", Vol. 3, Issue 1, pp. 500-504, March 2012.

[18] Zhang X. et al, "Time-frequency scaling transformation of the phonocardiogram based on the matching pursuit method", IEEE Transactions on Biomedical Engineering, 45(8):972-9, 1998.

[19] T. H. Hung et. al., "Time-frequency analysis of heart sound signals based on Hilbert-Huang Transformation", IEEE 16th International Symposium on Consumer Electronics (ISCE), Harrisburg, PA; 1-3, June 2012.

[20] J. Gao et al., "Reference empirical mode decomposition", IEEE 27th Canadian Conference on Electrical and Computer Engineering (CCECE);1-4; May 2014.

[21] K. Schiecke et al , "Empirical mode decomposition of heart rate variability. A methodological study", IEEE 8th Conference of the European Study Group on Cardiovascular Oscillations (ESGCO);105-106, May 2014.

\section{BIOGRAPHIES}

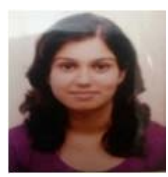

Mehak Saini (DOB: 1 April 1994) is B.Tech (ECE) and pursuing M.Tech from Deenbandhu Chhoturam University of Science and Technology Murthal, Sonepat (Haryana), India. She is a young Technocrat and Researcher. She has published 6 research papers in National/ International Journals. Her area of Interest is Optical Communication and Advanced Communication System.

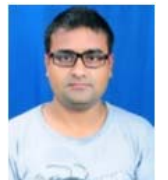

Mr. Madhwendra Nath (DOB:15 Jul 1985) is B.Tech., M.Tech from N.I.T. Jalandhar (Punjab), India. Currently, He is working as an Assistant Professor in Hindu college of Engineering Sonepat, Haryana, India. His area of Interest is Signal Processing and image Biometric Security. He has published various research papers in National, International and IEEE international conferences.

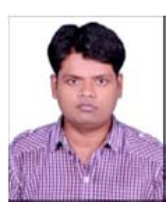

Mr. Priyanshu Tripathi (DOB: 17 March 1987 ) is B.Tech., M.Tech. from N.I.T. Jalandhar (Punjab), India. He is a young Technocrat and researcher. Currently, He is working as an Assistant Professor in Hindu college of Engineering Sonepat, Haryana, India. His area of Interest is Robotics 
and image processing. He has published various research papers in National, International and IEEE international conferences.

Dr. Sanju Saini is B.Tech.., M.Tech. \& PhD in Electrical Engineering. Currently, She is Assistant Professor in Deenbandhu Chhoturam University of Science and Technology Murthal, Sonepat ,Haryana, India. His area of interest is Control Syatem, Chaos based Nonlinear Dynamic System and Artificial Neural Networks. She has published more than 30 research papers in various reputed National and International journals and conferences. She has guided Dissertation of more than 20 M.Tech. Students

Prof.(Dr.) Kamalesh Kumar Saini is B.E., M.Tech. \& PhD in Electronics \& Communication Engineering. Currently, $\mathrm{He}$ is Director-Principal of Hindu College of Engineering Sonepat, Haryana, India. His Research area and area of interest is Optical Communication, Chaos based comm., Satellite Communication and Reliability Engineering. He has published more than 500 research papers in various reputed international journals, international conferences and IEEE International Transactions. He has guided Dissertation of more than 100 M.Tech. students and $7 \mathrm{Ph} . \mathrm{D}$. scholars.For further details, refer his website: www.drkksaini.in 